\title{
Overexpression of the CmJAZ1-like gene delays flowering in Chrysanthemum morifolium
}

\author{
Yunxiao Guan', Lian Ding', Jiafu Jiang $\mathbb{D}^{1}$, Yuanyue Shentu', Wenqian Zhao', Kunkun Zhao', Xue Zhang ${ }^{1}$,
} Aiping Song ${ }^{1}$, Sumei Chen ${ }^{1}$ and Fadi Chen ${ }^{1}$

\begin{abstract}
Chrysanthemum (Chrysanthemum morifolium) is one of the four major cut-flower plants worldwide and possesses both high ornamental value and cultural connotation. As most chrysanthemum varieties flower in autumn, it is costly to achieve annual production. JAZ genes in the TIFY family are core components of the jasmonic acid (JA) signaling pathway; in addition to playing a pivotal role in plant responses to defense, they are also widely implicated in regulating plant development processes. Here, we characterized the TIFY family gene CmJAZ1-like from the chrysanthemum cultivar 'Jinba'. CmJAZ1-like localizes in the nucleus and has no transcriptional activity in yeast. Tissue expression pattern analysis indicated that CmJAZ1-like was most active in the root and shoot apex. Overexpressing CmJAZ1-like with Jas domain deletion in chrysanthemum resulted in late flowering. RNA-Seq analysis of the overexpression lines revealed some differentially expressed genes (DEGs) involved in flowering, such as the homologs of the flowering integrators FT and SOC1, an FUL homolog involved in flower meristem identity, AP2 domain-containing transcription factors, MADS box genes, and autonomous pathway-related genes. Based on KEGG pathway enrichment analysis, the differentially transcribed genes were enriched in carbohydrate metabolic and fatty acid-related pathways, which are notable for their role in flowering in plants. This study preliminarily verified the function of CmJAZ1-like in chrysanthemum flowering, and the results can be used in molecular breeding programs aimed at flowering time regulation of chrysanthemum.
\end{abstract}

\section{Introduction}

Thines et al. ${ }^{1}$ performed exogenous jasmonic acid (JA) treatment of the Arabidopsis mutant opr3, and eight proteins containing the conserved ZIM domain were significantly induced, which led to the discovery of JAZ repressor proteins ${ }^{1,2}$. JAZs belong to the plant-specific TIFY family and possess three conserved domains: NT, $\mathrm{ZIM}$, and $\mathrm{Jas}^{3}$. The $\mathrm{N}$-terminus of the protein contains a weakly conserved NT domain, which can interact with the DELLA protein and the flowering repressor TARGET OF EAT1 (TOE1) ${ }^{4,5}$. The ZIM domain consists of 36 amino acids, including the conserved TIFY motif (TIF [F/Y] XG), which is essential for the formation of homo- or

Correspondence: Fadi Chen (chenfd@njau.edu.cn)

${ }^{1}$ State Key Laboratory of Crop Genetics and Germplasm Enhancement, Key Laboratory of Landscaping, Ministry of Agriculture and Rural Affairs, College of Horticulture, Nanjing Agricultural University, Nanjing 210095, China heterodimers among JAZ proteins and determines the combination of JAZ and NINJA in the JAZ-NINJA-TPL repressor complex ${ }^{6,7}$. The $\mathrm{C}$-terminal Jas domain is highly conserved, consisting of 12-29 amino acids, and controls interactions with the F-box protein CORONATINE INSENSITIVE1 (COI1 $)^{8,9}$. The function of JAZs in the JA signaling pathway has been revealed in Arabidopsis. The F-box protein COI1 perceives JA-Ile and forms the E3 ubiquitin ligase $\mathrm{SCF}^{\mathrm{COI} 1}$, which further targets jasmonateZIM domain (JAZ) proteins for degradation through the $26 \mathrm{~S}$ proteasome. Consequently, the transcription factors that are bound and repressed by JAZs are released, thereby regulating plant developmental processes and defense responses ${ }^{1,10,11}$.

Flowering at the suitable time is crucial for prosperous reproduction and has important commercial value for ornamental plants. The transformation of flowering plants 
from vegetative growth to reproductive growth is coordinated by a series of signal transduction pathways and sophisticated gene networks. Thus far, it has been proven that the photoperiod, vernalization, gibberellin, aging, autonomous, and ambient temperature pathways are involved in regulating the flowering time of Arabidopsis $^{12}$. Among them, the autonomous, age, and gibberellin pathways regulate flowering time through endogenous signals, which are closely related to the growth and development status of the plant itself, whereas the photoperiod and vernalization pathways respond to external environmental stimuli ${ }^{13}$. These six predominant pathways ultimately gather the common downstream floral integrators FLOWERING LOCUS T (FT) and SUPPRESSOR OF OVEREXPRESSION OF CO1 (SOC1), which can activate the expression of floral meristem identity genes, such as APETALA1 (AP1), LEAFY (LFY), and FRUITFULL $(F U L)$, and consequently lead to flowering ${ }^{14}$.

As pivotal components of the JA signaling pathway, JAZs not only play a crucial role in plant responses to environmental stresses and biotic challenges ${ }^{10,15,16}$ but also are widely implicated in the regulation of plant development processes, such as root growth ${ }^{17,18}$, leaf senescence $^{19}$, trichome initiation ${ }^{20}$, anthocyanin accumulation $^{20}$, flower abscission ${ }^{21}$, stamen development ${ }^{22}$, spikelet development ${ }^{23}$, and seed production ${ }^{24}$. To date, few studies have reported the relationship between JAZs and flowering regulation. Zhai et al. ${ }^{5}$ revealed that JAZ proteins (JAZ1, JAZ3, JAZ4, and JAZ9) could interact with the AP2 family proteins TOE1 and TOE2 and confirmed that JAZ1 can reduce the transcriptional inhibitory effect of TOE1 on $F T$ through interaction. Consequently, plants with AtJAZ1DJas overexpression exhibited early flowering ${ }^{5}$. The jaz7-1D mutant acquired by T-DNA insertion of the JAZ7 gene in the promoter led to earlier flowering than that in wild-type plants under short-day conditions ${ }^{25}$. Oblessuc et al. ${ }^{26}$ found that the mutant jaz41 exhibited delayed flowering and that overexpression of the JAZ4 protein, which deletes the Jas domain, accelerated flowering compared to that in the wild type. In addition to the differences between the wild type and transgenic plants in leaf initiation, plant height, and trichomes, overexpression of SIJAZ2 in tomato also led to advanced flowering transition ${ }^{27}$.

Chrysanthemum (Chrysanthemum morifolium) is a popular ornamental plant with high commercial value worldwide, and most chrysanthemum varieties flower in autumn. Therefore, it is essential to conduct research related to the regulation of chrysanthemum flowering time to achieve annual production. Although the role of JAZ proteins has been extensively studied in Arabidopsis, to the best of our knowledge, their functions in chrysanthemum have not been characterized. In this study, we isolated and characterized a TIFY family gene,
CmJAZ1-like. The transgenic lines overexpressing CmJAZ1-like without the Jas domain delayed flowering in C. morifolium. RNA-Seq analysis indicated that genes associated with flowering were differentially expressed. KEGG pathway enrichment analysis showed that a total of 1463 differentially expressed genes (DEGs) were enriched in carbohydrate metabolism and fatty acid-related pathways that are crucial for floral induction in plants ${ }^{28,29}$. Taken together, the results of this study link CmJAZ1-like to flowering time through regulation of the genes related to flowering and the metabolic processes of carbohydrates and fatty acids in chrysanthemum.

\section{Results}

Isolation and sequence analyses of CmJAZ1-like

As a gene encoding a protein that interacts with the positive regulator of chrysanthemum petal elongation CmTCP20, CmJAZ1-like was previously isolated from 'Jinba' chrysanthemum ${ }^{30}$ and comprises a 657-bp open reading frame (ORF) that encodes a 219-amino-acid polypeptide. CmJAZ1-like belongs to the TIFY protein family and has three conserved domains. As shown in Fig. 1A, the first conserved domain, the NT domain, is located at the $\mathrm{N}$-terminus and exhibits weak conservation; the second is the TIFY domain, which is between 313 and $411 \mathrm{bp}$ in size; and the last is the Jas domain, which is strongly conserved between 523 and $597 \mathrm{bp}$ and is crucial

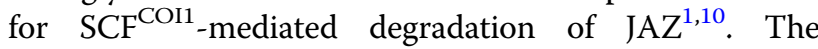
obtained phylogenetic tree revealed that CmJAZ1-like was highly homologous with the Artemisia annua AaJAZ1 protein (Fig. 1B), so this gene was named CmJAZ1-like. Amino acid sequence alignment indicated that the amino acid sequence similarity between CmJAZ1-like and homologous proteins in other species was $87.78 \%$ (AaJAZ1), 30.95\% (NtJAZ1), 30.04\% (AtJAZ1), 19.07\% (ZmJAZ4), and $31.50 \%$ (SlJAZ2).

\section{Subcellular localization, transcriptional activation, and expression patterns of CmJAZ1-like}

To investigate the subcellular localization of CmJAZ1like, the control vector 35S::GFP and the constructed vector 35S::GFP-CmJAZ1-like were introduced into onion epidermal cells via particle bombardment. In the transformed cells, red fluorescent protein was utilized as the nuclear marker (D53-mCherry); the green fluorescent protein (GFP) fluorescence of the control vector was observed in both the cytoplasm and the nucleus, while the GFP fluorescence of the constructed vector 35S::GFPCmJAZ1-like was detected in only the nucleus of the onion epidermal cells (Fig. 2A). These results suggested that CmJAZ1-like localizes in the nucleus.

To further examine the transcriptional activation of CmJAZ1-like, the gene was fused to the GAL4-binding domain (BD) and expressed in the yeast strain $\mathrm{Y} 2 \mathrm{H}$. 

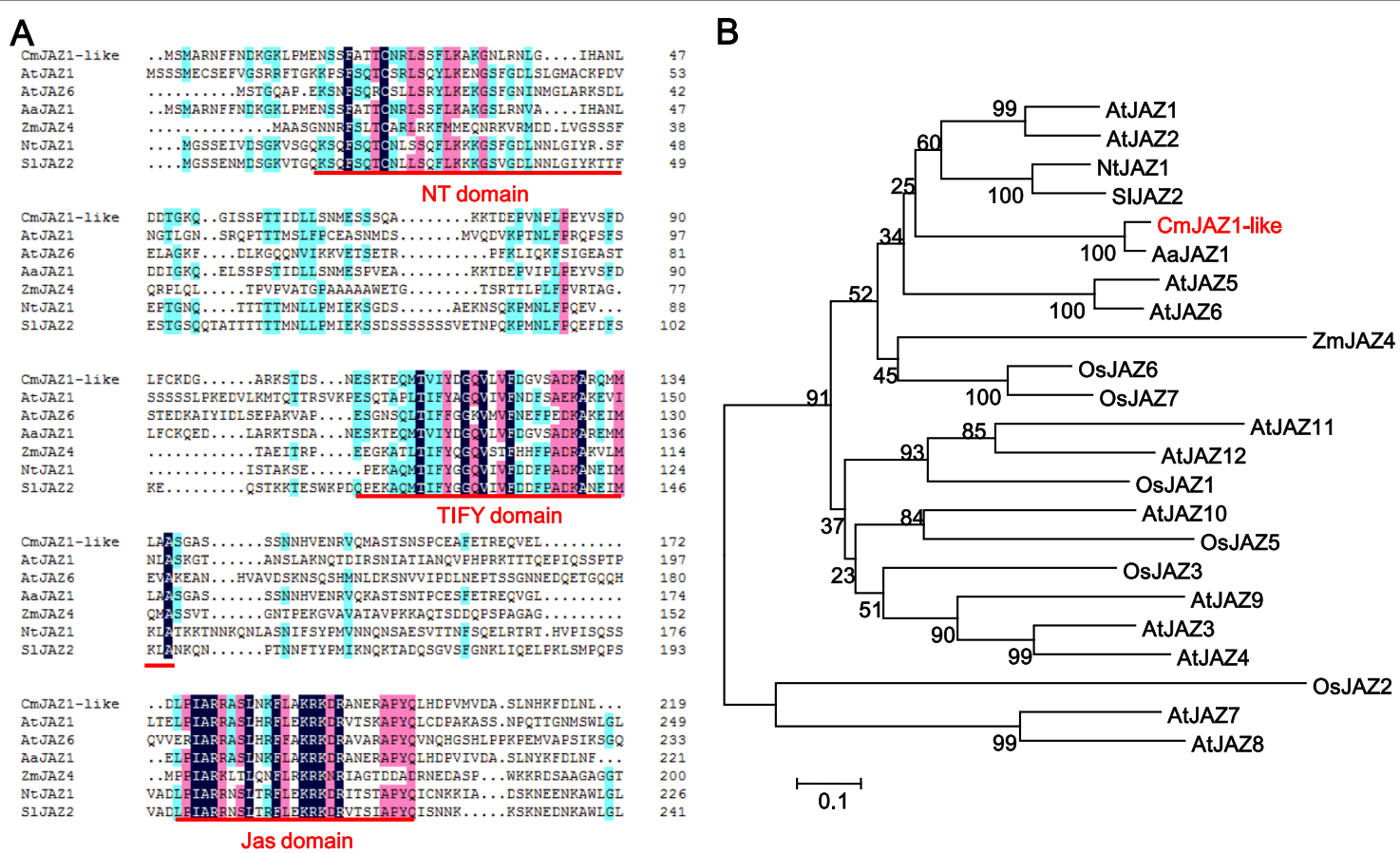

Fig. 1 Amino acid sequence analysis and phylogenetic tree of JAZ proteins. A Polypeptide sequence alignment of CmJAZ1-like and homologous proteins from other species. Navy blue represents 100\% identity, pink represents $75 \%$ identity, and light blue represents $50 \%$ identity. B Phylogenetic tree of CmJAZ1-like and related JAZ proteins from other species using the neighbor-joining method. The bar (0.1) indicates the branch length. The gene accession numbers are as follows: Arabidopsis thaliana AtJAZ1 (At1g19180), AtJAZ2 (At1g74950), AtJAZ3 (At3g17860), AtJAZ4 (At1g48500), AtJAZ5 (At1g17380), AtJAZ6 (At1g72450), AtJAZ7 (At2g34600), AtJAZ8 (At1g30135), AtJAZ9 (At1g70700), AtJAZ10 (At5g13220), AtJAZ11 (At3g43440), AtJAZ12 (At5g20900), Oryza sativa OsJAZ1 (XP_015635690.1), OsJAZ3 (XP_015651050.1), OsJAZ2 (XP_015646242.1), OsJAZ5 (XP_015634258.1), OsJAZ6 (XP_015630632.1), OsJAZ7 (XP_015647536.1), Artemisia annua AaJAZ1 (PWA58763.1), Zea mays ZmJAZ4 (XP_008664988), Nicotiana tabacum NtJAZ1 (BAG68655.1), and Solanum lycopersicum SIJAZ2 (NP_001234883.1)

The $\mathrm{pCL} 1$ vector served as the positive control, while the pGBKT7 vector was the negative control. The results showed that yeast cells harboring the $\mathrm{pCL1}$ vector grew extremely well on SD/-Ade-His medium and could turn blue on $\mathrm{SD} /$-Ade-His medium coated with X- $\alpha$-gal, whereas those harboring the pGBKT7 vector and the constructed pGBKT7-CmJAZ1-like vectors were unable to grow on $\mathrm{SD} /$-Ade-His medium, which suggested that CmJAZ1-like exhibited no transcriptional activation (Fig. 2B).

The expression pattern of CmJAZ1-like in different tissues of 'Jinba' chrysanthemum plants was determined via $\mathrm{qRT}-\mathrm{PCR}$. The data showed that CmJAZ1-like was most abundantly transcribed in the root, followed by the shoot apex, leaf, and flower, with only a low level of transcript detectable in the stem (Fig. 2C).

\section{Overexpression of CmJAZ1-like $\Delta$ Jas delays flowering in $C$. morifolium}

The Jas domain of JAZ proteins is essential for $\mathrm{SCF}^{\mathrm{COI1}}$. dependent proteasome degradation through interactions with $\mathrm{COI} 1^{1,31}$. Overexpression of the sequence with Jas domain deletion or mutation has been widely used to study the function of $J A Z$ genes $^{5,26,32}$. To further investigate the biological function of CmJAZ1-like in chrysanthemum, we transformed the CaMV $35 \mathrm{~S}$ promoter followed by the coding sequence (CDS) of CmJAZ1-like without the Jas domain into 'Jinba' chrysanthemum through Agrobacterium-mediated leaf disc transformation, obtaining eight 35Spro:CmJAZ1-like $\triangle$ Jas overexpression lines that were validated by PCR at the DNA level and by qRT-PCR analysis during the tissue culture period (Fig. S1). We then transplanted three representative CmJAZ1DJas-overexpressing transformants (OX-\#3, OX-\#1, and OX-\#2) to the field for further phenotypic observation. These three positive transgenic lines were reconfirmed through RT-PCR analysis by using specific primers designed using the GFP tag as a forward primer and the gene CDS as a reverse primer, and their transcriptional levels were measured by qRT-PCR using CmJAZ1-like-specific primers (Fig. 3A).

We observed that the flower buds of transgenic plants emerged later than those of the wild-type plants under natural conditions. At $63 \mathrm{~d}$ after transplantation, flower buds were found in most of the wild-type plants, while this did not occur in the CmJAZ1DJas-OX-\#3 and 


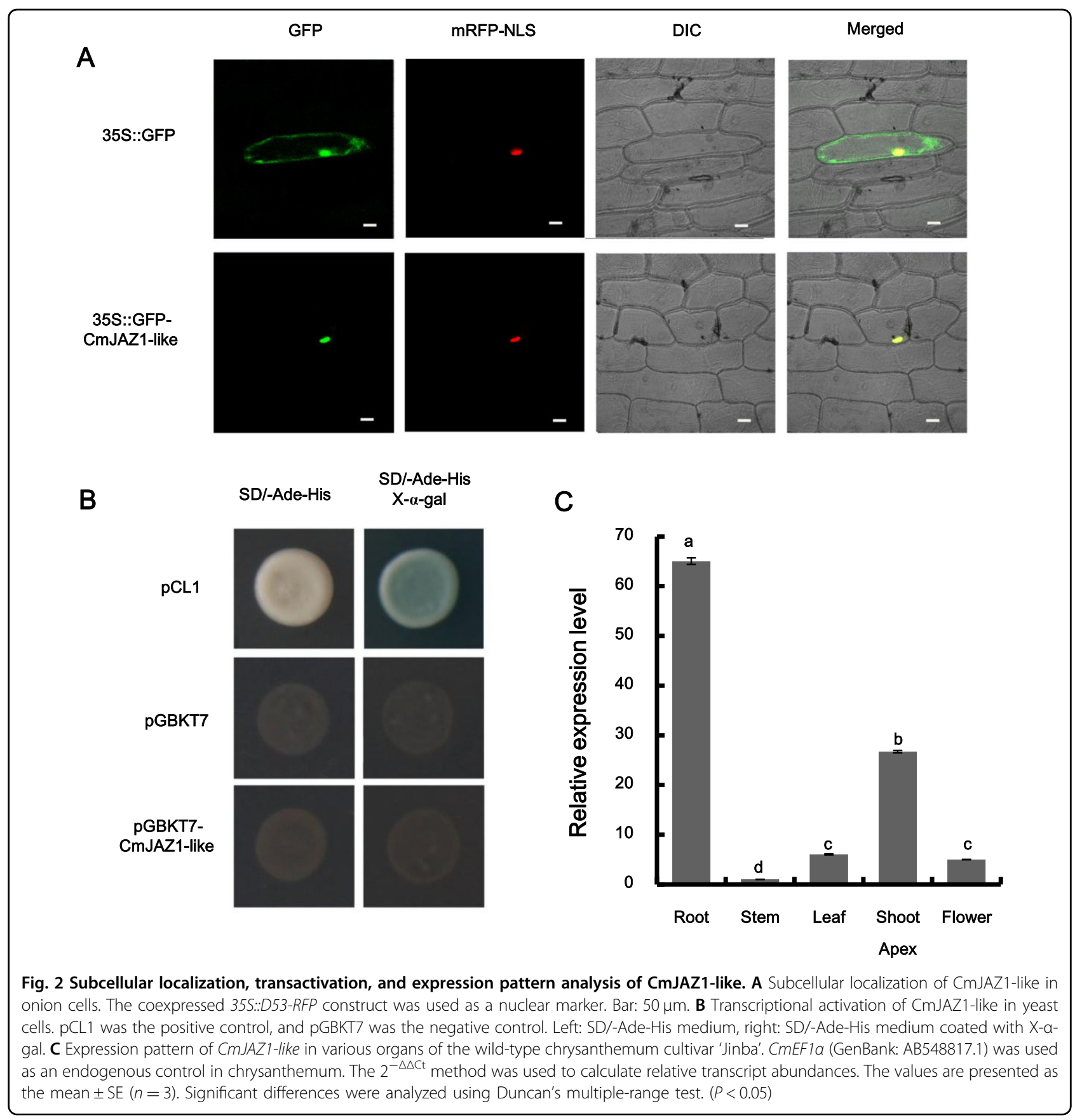

CmJAZ1DJas-OX-\#1 plants. Moreover, at $91 \mathrm{~d}$ after transplantation, the wild-type plants had entered the visible color stage, while the CmJAZ1DJas-OX-\#3 and CmJAZ1DJas-OX-\#1 plants were still at the flower bud development stage (Fig. 3B, C), and at $104 \mathrm{~d}$ after planting, the wild-type plants were already exhibiting flower

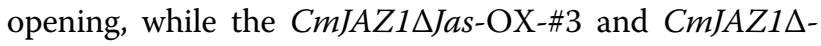
Jas-OX-\#1 plants were at the visible color stage (Fig. 3D). Subsequently, the time when flower buds emerged and bloomed in the CmJAZ1DJas-OX-\#3 and CmJAZ1DJasOX-\#1 plants was $12 \mathrm{~d}$ later than that in the wild-type plants. Furthermore, the difference in flowering time observed between CmJAZ1DJas-OX-\#2 and the wild-type plants was not as apparent (only $4 \mathrm{~d}$ ). The severity of the phenotype was positively correlated with the expression level of the transgenic lines. These results indicate that CmJAZ1-like has the capacity to regulate flowering time in chrysanthemum. 


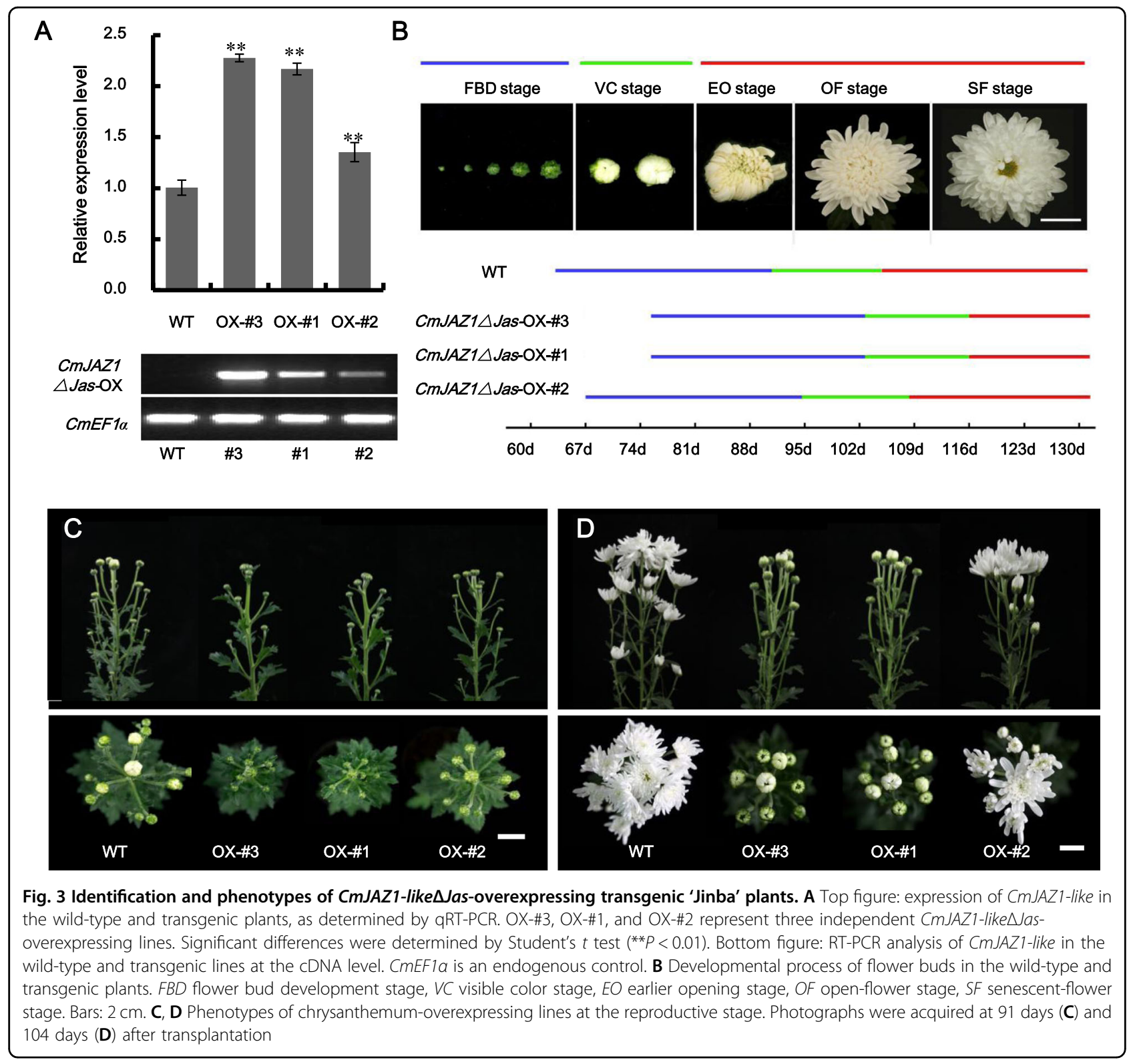

Transcriptome sequencing analysis and functional enrichment of DEGs in CmJAZ1-like $\mathrm{Cas}$ overexpression lines

To better understand the regulatory mechanisms of CmJAZ1-like involved in the regulation of flowering time, RNA-seq analysis was performed. RNA extracted from the seventh unfolded leaf of the wild-type and CmJAZ1DJas-OX-\#3 plants was used as the RNA-Seq sample. A total of $390.5 \mathrm{M}$ clean reads were generated from six samples (three replicates each for the wild-type and transgenic lines), with each sample producing a minimum of $62.66 \mathrm{M}$ clean reads. After implementing the assembly procedure, we obtained 111,669 unigene sequences with a mean length of $1159 \mathrm{bp}$; the N50 was 1703 bp (Table S1). The Pearson's correlation coefficient of the three wild-type samples ranged from 0.989 to 1 , and that of the CmJAZ1DJas-OX-\#3 samples ranged from 0.982 to 1 (Fig. $4 \mathrm{~A}$ ), which indicated that the transcript abundances of the biological replicate samples were highly correlated. The DEseq2 method was used to identify $\mathrm{DEGs}^{33}$. A total of 4204 DEGs were obtained between the wild-type and CmJAZ1DJas-OX-\#3 with a $\mathrm{Q}$ value (adjusted $P$ value) $\leq 0.05$ as the standard. Among these genes, 2122 were upregulated, and 2082 were downregulated (Fig. 4B; Table S3).

Among the 4204 DEGs, a total of 1463 possessed KEGG annotations. To explore the functional categories and main biological pathways involving these DEGs, according to KEGG pathway annotation classification, we used the 


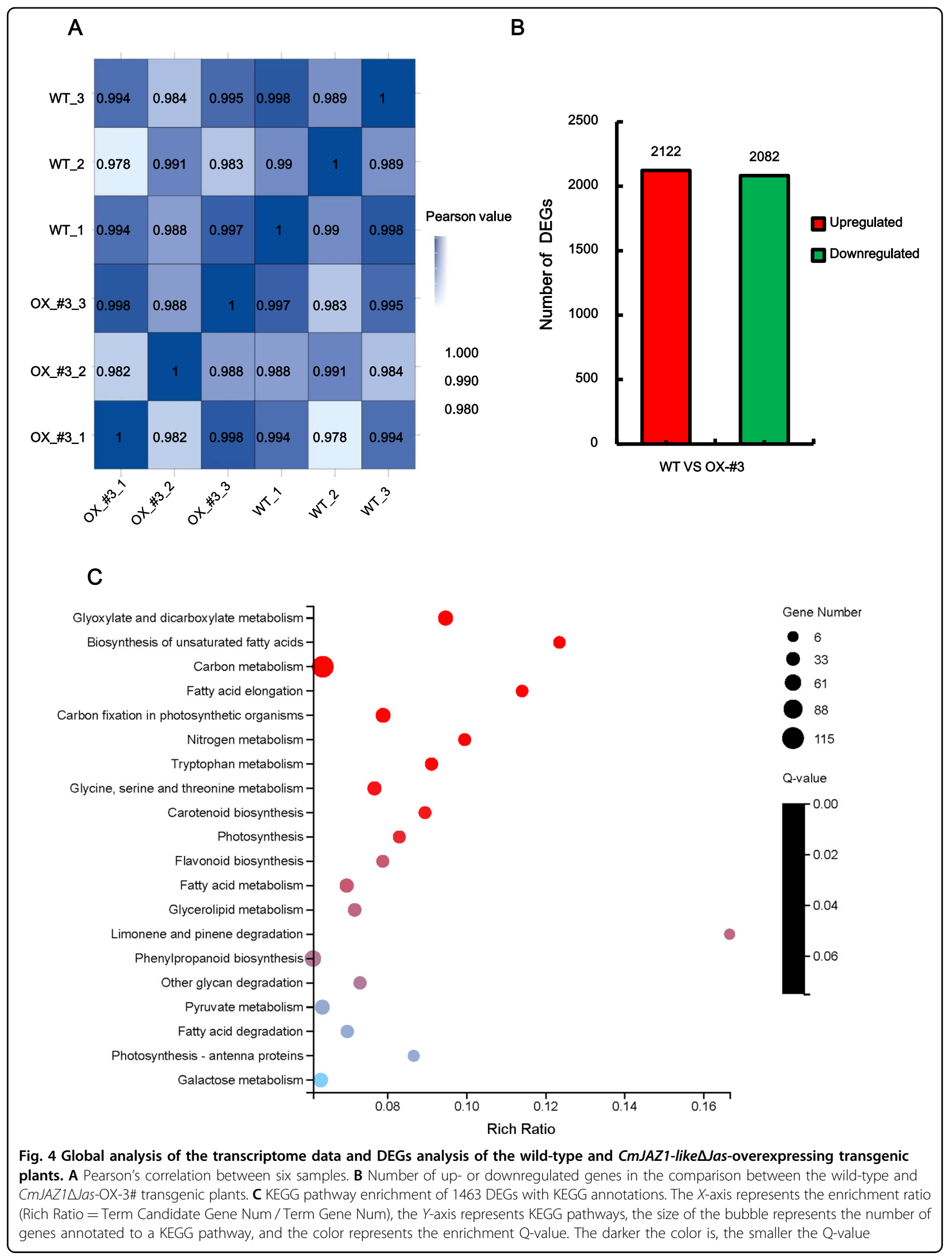


Table 1 DEGs related to flowering time between the wild-type and CmJAZ1-likeDJas-overexpressing transgenic plants

\begin{tabular}{lllll}
\hline Gene_ID & Annotation & log2(OX-JAZ/WT) & Q-value & Function \\
\hline CL2913.Contig4_All & SOC1 & -2.41 & 0.03 & Flowering integrator \\
CL3262.Contig5_All & FTL & -1.37 & $8.42 \mathrm{E}-14$ & Flowering integrator \\
Unigene36566_All & TEM1 & 0.55 & 0.009 & AP2 domain transcription factor \\
Unigene26493_All & TOE3 & 5.40 & $2.38 \mathrm{E}-24$ & AP2 domain transcription factor \\
CL14305.Contig7_All & FLC & 1.15 & 0.016 & MADS box gene \\
CL7056.Contig2_All & SVP & 1.24 & $6.95 \mathrm{E}-07$ & MADS box gene \\
CL10079.Contig1_All & FUL & -1.35 & $4.93 \mathrm{E}-12$ & MADS box gene \\
CL2797.Contig1_All & RVE1 & -0.67 & 0.0003 & Photoperiod pathway \\
Unigene1508_All & ELF4 & 0.59 & 0.009 & Photoperiod pathway \\
CL4634.Contig9_All & DRM1 & -2.24 & 0.0006 & Autonomous pathway \\
CL1053.Contig1_All & FVE & -2.78 & $5.03 \mathrm{E}-26$ & Autonomous pathway \\
\hline
\end{tabular}

Significant differences were determined with $\mathrm{Q}<0.05$ and $|\log 2(\mathrm{OX}-\mathrm{JAZ} / \mathrm{WT})|>0.5$ WT wild type

phyper function in $\mathrm{R}$ software for enrichment analysis and selected the 20 KEGG terms with the lowest $Q$ values as a showcase (Fig. 4C). Most of these transcripts were involved in metabolic pathways. These metabolic pathways included five carbohydrate metabolic pathways and five fatty acidrelated pathways. Carbohydrates play a crucial role in floral induction as the signal and energy supply factors ${ }^{34,35}$. In our study, a maximum of 115 genes were annotated to carbon metabolism, and a total of 47 candidate genes were enriched to glyoxylate and dicarboxylate metabolism. Moreover, 45 genes were involved in carbon fixation in photosynthetic organisms; the glycan degradation and galactose metabolism KEGG terms contained 28 and 42 genes, respectively. In addition, five fatty acid-related pathways, including "Biosynthesis of unsaturated fatty acids", "Fatty acid elongation", "Fatty acid metabolism", "Glycerolipid metabolism", and "Fatty acid degradation", were also enriched. It has been reported that fatty acids are involved in flowering regulation $^{36}$. These results indicate that carbohydrates and fatty acids may be the cause of the difference in flowering time between the transgenic and wild-type plants. Pathways such as "Tryptophan metabolism", "Glycine, serine, and threonine metabolism", "Photosynthesis", and "Photosynthesisantenna proteins", which are related to flowering, were also represented. In addition to the difference in flowering time, we also observed growth-restricted roots and smaller leaves in the CmJAZ1-like $\Delta$ Jas overexpression plants compared to the wild type. These may also be the result of changes in metabolic pathway-related genes.

\section{Flowering-related genes were differentially expressed in CmJAZ1-like Das overexpression lines}

Given that overexpression of CmJAZ1-like with the Jas domain deleted delays flowering, we focused on the DEGs between CmJAZ1DJas-OX-\#3 and wild-type plants that were implicated in the flowering pathways (Table 1). According to the annotation, we found that the homologs of the flowering integrators FT (CL3262.Contig5_All) and SOC1 (CL2913.Contig4_All) were significantly decreased in the CmJAZ1DJas-OX-\#3 lines. FUL has been reported as a positive regulator of flower meristem identity ${ }^{37}$, and its homologous gene (CL10079.Contig1_All) was also downregulated, whereas homologs of the MADS box genes FLC (CL14305.Contig7_All) and SVP (CL7056.Contig2_All), which act as flowering inhibitors, were upregulated in CmJAZ1DJas-OX-\#3 compared to that in the wild-type strain. In addition, two AP2 domain-containing transcription factors, namely, CmTOE3 (Unigene26493_All) and CmTEM1 (Unigene36566_All), homologs of which in Arabidopsis can repress the expression of the florigen $F T^{38}$, were also more abundant in CmJAZ1 1 Jas-OX-\#3. Notably, the homologs of DRM1 (CL4634.Contig9_All) and FVE (CL1053.Contig1_All), which are involved in autonomous pathways, were significantly reduced. We also observed that the homologous genes of the rhythm regulators $R V E 1$ (CL2797.Contig1_All) and ELF4 (Unigene1508_All) were mildly altered.

To verify the authenticity of the expression levels obtained from the transcriptome data, we selected some of the genes related to flowering time above and detected their transcripts using real-time quantitative PCR. In 'Jinba' chrysanthemum, the expression of the elongation factor $1 \alpha(C m E F 1 \alpha)$ gene was used as an internal control. The templates for qRT-PCR were sourced from the seventh unfolded leaf of the wild type and the transgenic lines CmJAZ1DJas-OX-\#3, CmJAZ1DJas-OX-\#1, and CmJAZ1DJas-OX-\#2. As shown in Fig. 5, the changes in transcript expression obtained by qRT-PCR were identical 


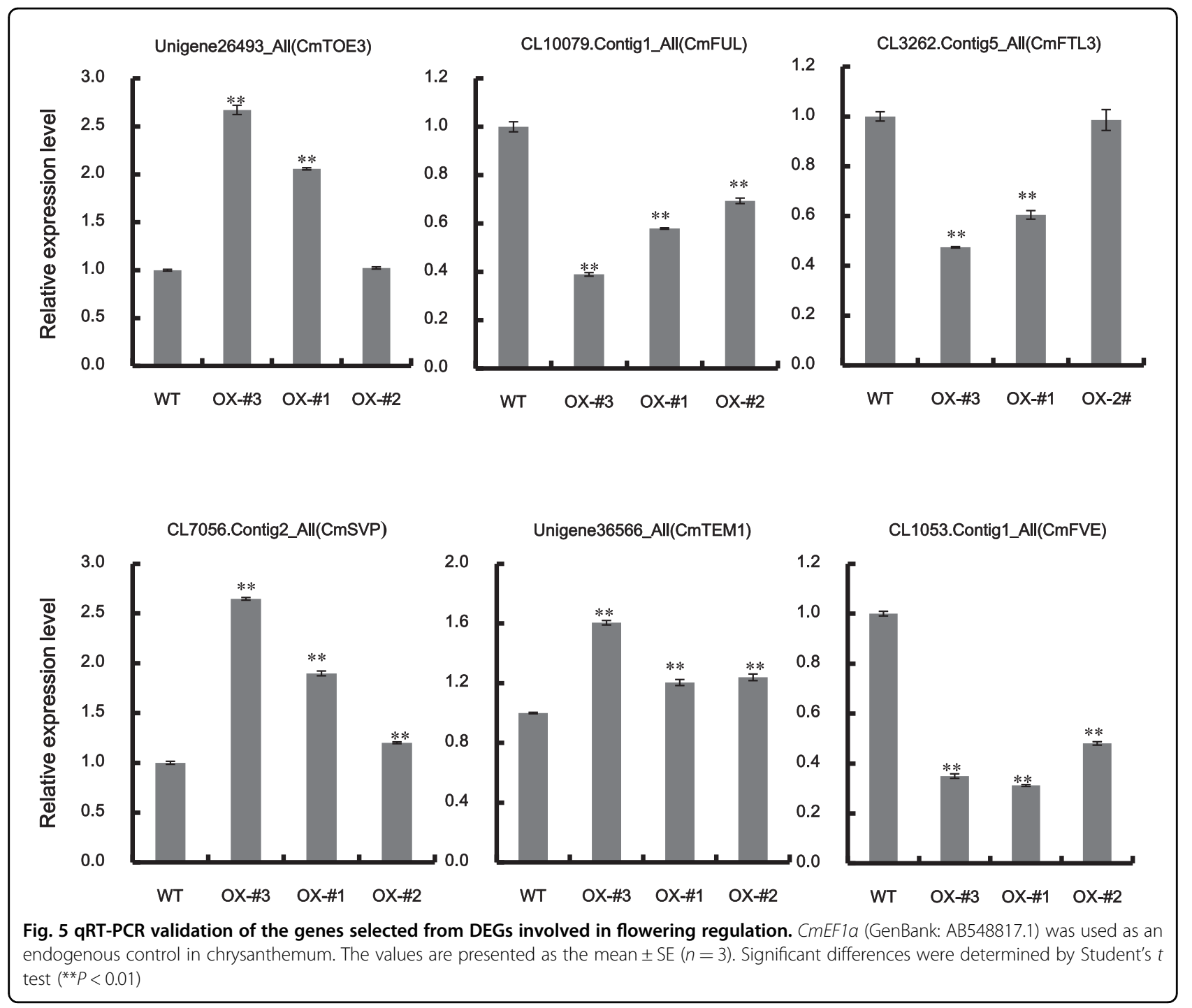

to those acquired by DEG expression profiling. Collectively, the above expression results suggest that the transcriptome data were credible and that CmJAZ1-like affects flowering time by regulating the genes related to flowering.

\section{Discussion}

As essential components of JA signal transduction, JAZ proteins not only inhibit the transcription of the JA signaling response but also interact with and repress other transcription factors to affect various signaling pathways and metabolic processes of plant growth ${ }^{39}$. To the best of our knowledge, there have been no studies on the function of TIFY family genes in chrysanthemum. Here, we identified the TIFY gene CmJAZ1-like, and transcription profiling indicated that CmJAZ1-like was abundantly expressed in the root and shoot apex. While expression in the shoot apex may be involved in flowering time control
(Fig. 2C), the most active expression was observed in the root, which may reveal an additional function of CmJAZ1like. Phylogenetic analysis and amino acid sequence alignment indicated that the CmJAZ1-like protein is closely related to another composite protein, AaJAZ1, with $87.78 \%$ sequence similarity, followed by the SlJAZ2 and AtJAZ1 proteins in another branch, the sequence similarities of which with CmJAZ1-like were only 31.50\% and $30.04 \%$, respectively (Fig. 1A, B). This revealed that the sequence and structure of JAZ proteins in different species listed in the phylogenetic tree, except Artemisia annua, displayed marked differences.

Flowering is a very complex process that is affected by endogenous developmental signals and external environmental factors ${ }^{13}$. Based on RNA-seq transcript profiling, we obtained 12 flowering-related homologous genes that were differentially expressed in CmJAZ1-like Jas-overexpressing transgenic chrysanthemum plants compared 
with wild-type plants (Table 1). FUL is redundant with $A P 1$ in regulating floral meristem identity ${ }^{40}$ and is also implicated in flowering ${ }^{41}$. Zhao et al. ${ }^{42}$ generated $p 35 S:$ : GFP-CmFL2 transgenic chrysanthemum plants that exhibited early flowering. CmFUL is a homologous gene of CmFL2 and was significantly downregulated in CmJAZ1-like $\Delta$ Jas transgenic plants. The transcription factors TEM1 and TOE3 containing the AP2 domain are repressors of the florigen $F T$. Zhai et al. ${ }^{5}$ revealed that JAZ1 could interact with TOE1 and thereby reduce the transcriptional inhibitory effect of TOE1 on $F T$, leading to early flowering. In this study, the expression levels of CmTOE3 and CmTEM1 were upregulated, whereas CmFTL3 displayed decreased expression. These results indicated that CmJAZ1-like can influence the AP2 domain transcription factors CmTOE3 and CmTEM1 at the transcriptional level in chrysanthemum. However, the regulatory mechanism between them requires further investigation. The autonomous pathway is an independent method for induction of flower formation that is not regulated by external signals. The autonomous pathway genes regulate $F T$ by repressing $F L C$ and $S V P$. FVE is a key regulator in this pathway; it binds to CLF-PRC2 via the encoded WD40 protein MSI4 to inhibit FLC expression $^{43}$. Zhu et al. ${ }^{44}$ confirmed that $d r m 1$ is a typical late flowering mutant and is most likely associated with the autonomous flowering pathway. Moreover, FLC had lower expression in drm1. As shown in Table 1, the expression levels of the CmDRM1 and CmFVE genes were significantly reduced, whereas those of CmFLC and CmSVP were highly increased, in CmJAZ1DJas-OX-\#3. These results showed that CmJAZ1-like-mediated regulation of flowering time depends partly on the autonomous pathway. Among these DEGs, the homologous genes of the rhythm regulators $R V E 1$ and ELF4, which are involved in the photoperiod pathway ${ }^{45}$, were mildly altered at the transcription level. Therefore, overexpression of the CmJAZ1-like gene caused increased expression of the AP2 transcription factors CmTOE3 and CmTEM1, reduced expression of autonomous pathway genes, and slight changes in the rhythm regulators CmRVE1 and CmELF4. All of these factors together induced the expression of the flowering inhibitors CmFLC and $C m S V P$, in addition to downregulating the integrators CmFTL3 and CmSOC1 and a positive regulator of flower meristem identity, resulting in delayed flowering ${ }^{46,47}$.

Previous studies have demonstrated that carbohydrates and fatty acids are essential for floral induction in plants $^{28,29}$. Through KEGG pathway enrichment analysis, the differentially transcribed genes between the transgenic and wild-type plants were enriched in carbohydrate metabolic and fatty acid-related pathways. Carbohydrates such as sucrose, glucose, and starch can provide energy for flower induction and are important signal transmission factors during this process ${ }^{34,35}$. The effect of fatty acids on flowering regulation has also been confirmed. In Arabidopsis, overexpressing a fatty acid amide hydrolase gene can accelerate flowering ${ }^{36}$. Furthermore, in a de novo transcriptome study of Dendrobium, a total of 84 flowerspecific expressed transcripts were also enriched in two fatty acid biosynthesis-related pathways ${ }^{48}$. Chen et al. ${ }^{49}$ reported that DEGs between two apple varieties with different flowering abilities were mainly involved in carbohydrate, fatty acid, and lipid pathways. This indicated that the delay in flowering caused by overexpressing CmJAZ1like may be ascribed to the changes in the carbohydrate and fatty acid pathways.

In the present study, overexpression of CmJAZ1like $\Delta$ Jas delayed flowering in C. morifolium. However, in Arabidopsis, AtJAZ1DJas overexpression in plants accelerated flowering compared to that in the wild type ${ }^{5}$. A similar situation was also observed in a study of the tomato SlJAZ2 gene, which is homologous to $A t J A Z 1^{27}$. In Arabidopsis, ectopic expression of the full-length JAZ1 cDNA did not lead to an early flowering phenotype; however, 35Spro:SlJAZ2 transgenic plants flowered one week earlier than the wild type $\mathrm{e}^{27}$. Furthermore, with overexpression of the R2R3 MYB transcription factor CmMYB2 in Arabidopsis and chrysanthemum, the transgenic plants exhibited an opposite phenotype in terms of flowering time ${ }^{50}$. These phenomena probably result from the differences in the sequence and structure of proteins in different species, which generate diverse protein characteristics and lead to the formation of different dimers with different proteins, which further participate in diverse regulatory pathways. In addition, Arabidopsis is a long-day plant, whereas the 'Jinba' chrysanthemum is a short-day plant. The photoperiod conditions and different day-length requirements for flowering may also be the cause of these discrepant phenotypes. However, the detailed mechanism leading to these differences requires further research.

In summary, we isolated a TIFY gene, CmJAZ1-like, from chrysanthemum and found clues that CmJAZ1-like functions in the chrysanthemum flowering process. Transcriptome sequencing revealed that flowering inhibitors, such as homologs of $F L C, S V P$, and AP2 domaincontaining transcription factors, were upregulated, while homologs of the flowering integrators $F T$ and SOC1, one $F U L$ homolog, and homologs of the autonomous pathway genes DRM1 and FVE were significantly downregulated. A total of 1463 DEGs with KEGG annotations were enriched in carbohydrate metabolic and fatty acid-related pathways. This study identified the function of CmJAZ1like in the flowering regulation of chrysanthemum, laying a foundation for developing molecular breeding programs aimed at flowering time regulation of chrysanthemum in the future. 


\section{Materials and methods}

\section{Plant materials and growth conditions}

In this study, we used a popular cut-flower chrysanthemum cultivar, 'Jinba', with white flowers, as the genetically modified material. Cuttings of the transgenic and wild-type plants were sourced from the Chrysanthemum Germplasm Resource Preserving Center, Nanjing Agricultural University (Nanjing, China). Four- to fiveleaf-stage rooted seedlings, including the transgenic lines and wild-type plants, were transplanted in a multispan greenhouse on July 25, 2019, in which the relative humidity was maintained at $70 \%$ and the day/night temperature was $34^{\circ} \mathrm{C} / 27^{\circ} \mathrm{C}$. The flowering time was observed under natural light cycle conditions, and the observation of the phenotype was repeated three times.

\section{Isolation of CmJAZ1-like}

Total RNA was extracted from snap-frozen flower buds of the 'Jinba' cultivar using RNAiso Plus reagent (Takara Bio, Tokyo, Japan), and $1 \mu \mathrm{g}$ of the RNA was used for reverse transcription amplification. First-strand cDNA was synthesized by M-MLV reverse transcriptase (Takara Bio). Based on the Unigene2375 sequence in the chrysanthemum 'Jinba' transcriptome ${ }^{51}$, the primer pair CmJAZ1-F/R was designed using Primer 5.0 software (www.bbioo.com/ Soft/2005/114.htm) to amplify an internal fragment of CmJAZ1-like (Table S2) using Phusion High-Fidelity DNA Polymerase (Thermo Fisher Scientific, Waltham, MA, USA). To check the CmJAZ1-like nucleotide sequence, the amplicon was inserted into the pMD19-T vector (Takara Bio) using solution I ligase (Takara Bio) for sequencing.

\section{Sequence analysis of CmJAZ1-like}

The amino acid sequences of JAZ proteins in Arabidopsis were downloaded from TAIR (http://www.arabidopsis.org/); the amino acid sequences of JAZ1 homologs in other species were acquired from GenBank (ww.ncbi.nlm.nih.gov). All of these sequences (Table S4) were subjected to phylogenetic analysis using MEGA 5.0 software based on the neighbor-joining method implemented with 1000 bootstrap replicates $^{52}$. Multiple-sequence alignment analysis of the JAZ proteins was implemented in DNAMAN software.

\section{Subcellular localization of CmJAZ1-like}

To understand the localization of the CmJAZ1-like protein in plant cells, we used the primer pair CmJAZ1pENTR1A-F/R (Table S2) with the restriction site to amplify the full-length ORF of the CmJAZ1-like gene. Then, the amplicons and the $\mathrm{pENTR}^{\mathrm{TM}} 1 \mathrm{~A}$ vector (Invitrogen, Carlsbad, CA, USA) were both restricted to Sal I and Not $I$ and ligated after digestion using solution I ligase (Takara Bio) to generate the pENTR1A-CmJAZ1-like vector. The constructs were then recombined with the pMDC43 vector via LR Clonase ${ }^{\mathrm{TM}}$ II (Invitrogen) to obtain the GFP fusion plasmid p35S::GFP-CmJAZ1-like. According to the Cold Spring Harbor Experiment Manual ${ }^{53}$, both the 35::GFP-CmJAZ1-like and 35::GFP plasmids were transiently introduced into onion (Allium cepa) epidermal cells by particle bombardment (PDS-1000; Bio-Rad Laboratories, Hercules, CA, USA). The fluorescence signal of the transformed cells was detected using a Zeiss LSM 780 confocal microscope (Carl Zeiss AG, Oberkochen, Germany) after incubation in the dark for $16 \mathrm{~h}$ at $22^{\circ} \mathrm{C}$ on Murashige and Skoog (MS) ${ }^{54}$ medium.

\section{Transcriptional activity analysis of CmJAZ1-like}

A yeast assay system (Takara Bio) was used to examine the transcriptional activity of CmJAZ1-like. An ORF of CmJAZ1-like lacking a termination codon was amplified using the primer pair CmJAZ1-BD-F/R (Table S2). Then, the amplicon and pGBKT7 vector (Invitrogen) were both digested with EcoRI and BamHI, and the PCR products were ligated using solution I ligase (Takara Bio), generating the construct pGBKT7-CmJAZ1-like. Following the manufacturer's protocol, the plasmids pCL1 (positive control), pGBKT7 (negative control), and pGBKT7-CmJAZ1-like were transformed into the yeast strain $\mathrm{Y} 2 \mathrm{H}$. Transformants containing pGBKT7-CmJAZ1-like or pGBKT7 were cultured on SD/-Trp medium, whereas those containing the positive control pCL1 were cultured on SD/-Leu medium. SD medium (a minimal, synthetic, defined medium) includes carbon sources, yeast nitrogen sources without amino acids, and dropout supplements, which can be added to the minimal SD base to make a synthetic, defined medium lacking the specified nutrients. After 3 days at $30^{\circ} \mathrm{C}$, we selected single clones and transferred them onto $\mathrm{SD} /$-Ade-

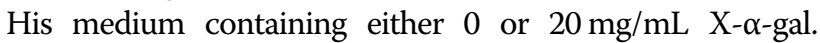
Similarly, after 3 days of growth, we assessed whether there were blue spots on the plates.

\section{CmJAZ1-like genetic transformation and phenotype observation}

The same method as that described in the previous subsection was used to construct the vector pENTR1ACmJAZ1-like $\Delta$ Jas. We used the primer pair CmJAZ1 $\Delta$ JaspENTR1A-F/R (Table S2) with a restriction site to generate the pENTR1A-CmJAZ1-like $\Delta$ Jas vector. The pENTR1ACmJAZ1-like $\Delta$ Jas construct was then recombined with the pMDC43 vector via an LR Clonase ${ }^{\mathrm{TM}}$ II (Invitrogen) reaction to obtain the plant expression vector pMDC43-CmJAZ1like $\Delta$ Jas. The construct 35S::CmJAZ1-like $\triangle$ Jas was then transformed into competent Agrobacterium EHA105 cells for genetic transformation of chrysanthemum. Then, the Agrobacterium was used to infect the leaf discs to complete the transformation ${ }^{30}$. Young leaves of 'Jinba' tissue culture plantlets aged 30 to 35 days were selected and cut into leaf discs of $0.3 \mathrm{~cm} \times 0.3 \mathrm{~cm}$ with wounds on the edges. Seedlings differentiated from infected leaf discs were transferred to MS 
medium containing $8 \mathrm{mg} / \mathrm{L}$ hygromycin for resistance screening. The forward primer GFP-F for the vector and the reverse primer for the ORF of the CmJAZ1-like gene (Table S2) were adopted to detect positive transgenic plants using RT-PCR. Subsequently, qRT-PCR was performed to assess the relative expression levels of the positive lines with the CmJAZ1-RT-F/R (Table S2) primers. Each sample was analyzed with three biological and three technical replicates. The wild-type and transgenic plants were cultivated in a greenhouse to observe their phenotypes, and each line contained 40 plants.

\section{RNA-Seq analysis}

The seventh unfolded leaf of CmJAZ1DJas-OX-\#3 and wild-type plants were sampled 64 days after transplantation. Each sample contained three biological replicates. The total RNA from snap-frozen samples was extracted using an RNA Isolation Kit (Waryong, Beijing, China) following the manufacturer's protocol and then subjected to Illumina sequencing at Beijing Genomics Institute (Shenzhen, China) using a BGISEQ-500 platform. Reads with low quality, joint contamination, and high content of unknown bases $(\mathrm{N})$ were filtered out from the original data to obtain clean reads ${ }^{55}$. The Trinity program was then utilized to conduct de novo assembly of clean reads, and Tgicl was used to cluster the assembled transcripts for redundancy to obtain unigenes ${ }^{56}$. The assembled unigenes were annotated using seven functional databases (KEGG, GO, NR, NT, SwissProt, Pfam, and KOG). We then used Bowtie2 to align clean reads to the reference gene sequence and RSEM to calculate the expression levels of the genes and transcripts $^{57}$. A Q-value below 0.05 was regarded as the threshold for DEGs ${ }^{58}$. Kyoto Encyclopedia of Genes and Genomes (KEGG) enrichment analyses of the annotated DEGs were performed on the BGI Interactive Reporting System (https://report.bgi.com/ps/login/login.html).

\section{Quantitative RT-PCR analysis}

For the expression profile analysis of CmJAZ1-like in different tissues, young adventitious roots, stems, leaves, and shoot apexes were harvested at the vegetative stage, and the outermost whorl of ray florets was collected at the reproductive stage. The chrysanthemum cultivar 'Jinba' used for sampling was cultivated in a greenhouse under natural light conditions. To verify the expression of DEGs excavated by transcriptome sequencing and related to flowering time between the transgenic and wild-type plants, the seventh unfolded leaf of CmJAZ1DJas-OX-3\#,

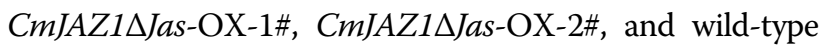
plants was sampled 64 days after transplantation. Each sample had three replicates, and total RNA was extracted using an RNA Isolation Kit (Waryong) as mentioned above. Subsequently, $1 \mu \mathrm{g}$ of total RNA was reverse transcribed using M-MLV reverse transcriptase (Takara Bio). qRT-
PCRs $(20 \mathrm{~mL}$ reaction mixture containing $10 \mu \mathrm{L}$ of SYBR Premix Ex Taq $^{\mathrm{TM}}$ II (Takara Bio)) were performed using a Roche LightCycler 96 real-time fluorescence quantitative PCR instrument (Roche, Basel, Switzerland). Primers used for qRT-PCR were designed online (https://www.ncbi.nlm. nih.gov/tools/primer-blast/) and are listed in Table S2. The

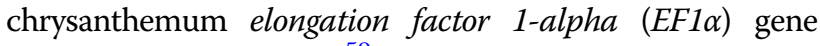
(GenBank: AB548817.1) ${ }^{59}$ was chosen as the reference. Each sample was evaluated using three biological and three technical replicates. The relative abundance of transcripts was analyzed using the $2^{-\Delta \Delta \mathrm{Ct}} \operatorname{method}^{60}$.

\section{Statistical analysis}

Significant differences among the different tissues were obtained by Duncan's multiple-range test $(P<0.05)$. Significant differences between the transgenic and wild-type plants were determined using Student's $t$ test. ${ }^{*} P<0.05$; ${ }^{* *} P<0.01$. All statistical analyses were conducted using SPSS v19.0 (SPSS Inc., Chicago, IL).

\section{Acknowledgements}

This work was supported by the National Key Research and Development Program of China (2018YFD1000400), the National Natural Science Foundation of China (31930100, 31701959), and a Project Funded by the Priority Academic Program Development of Jiangsu Higher Education Institutions.

\section{Author contributions}

F.C., J.J., and S.C. supervised the project. Y.G. and L.D. conceived and designed the experiments. Y.G., Y.S., W.Z., K.Z., X.Z., and A.S. performed the experiments. Y.G. wrote the paper. L.D. and F.C. revised the paper. All authors read and approved the final paper.

\section{Conflict of interest}

The authors declare no competing interests.

Supplementary information The online version contains supplementary material available at https://doi.org/10.1038/s41438-021-00525-y.

Received: 17 November 2020 Revised: 23 January 2021 Accepted: 1 March 2021

Published online: 01 April 2021

\section{References}

1. Thines, B. et al. JAZ repressor proteins are targets of the SCF (COl1) complex during jasmonate signalling. Nature 448, 661-665 (2007).

2. Mandaokar, A. et al. Transcriptional regulators of stamen development in Arabidopsis identified by transcriptional profiling. Plant J. Cell Mol. Biol. 46, 984-1008 (2006).

3. Bai, Y., Meng, Y., Huang, D., Qi, Y. \& Chen, M. Origin and evolutionary analysis of the plant-specific TIFY transcription factor family. Genomics $\mathbf{9 8}$, 128-136 (2011)

4. Kazan, K. \& Manners, J. M. JAZ repressors and the orchestration of phytohormone crosstalk. Trends Plant Sci. 17, 0-31 (2012).

5. Zhai, Q. et al. Transcriptional mechanism of jasmonate receptor COl1mediated delay of flowering time in Arabidopsis. Plant cell 27, 2814-2828 (2015).

6. Acosta, I. F. et al. Role of NINJA in root jasmonate signaling. Proc. Natl Acad. Sci. USA 110, 15473-15478 (2013).

7. Pauwels, L. et al. NINJA connects the co-repressor TOPLESS to jasmonate signalling. Nature 464, 788-791 (2010).

8. Melotto, M. et al. A critical role of two positively charged amino acids in the Jas motif of Arabidopsis JAZ proteins in mediating coronatine- and jasmonoyl 
isoleucine-dependent interactions with the COl1 F-box protein. Plant J.: cell Mol. Biol. 55, 979-988 (2008).

9. Sheard, L. B. et al. Jasmonate perception by inositol-phosphate-potentiated COl1-JAZ co-receptor. Nature 468, 400-405 (2010).

10. Chini, A. et al. The JAZ family of repressors is the missing link in jasmonate signalling. Nature 448, 666-671 (2007).

11. Yan, J. et al. The Arabidopsis CORONATINE INSENSITIVE1 protein is a jasmonate receptor. Plant Cell 21, 2220-2236 (2009).

12. Srikanth, A. \& Schmid, M. Regulation of flowering time: all roads lead to Rome. Cell. Mol. Life Sci. 68, 2013-2037 (2011).

13. Fornara, F., de Montaigu, A. \& Coupland, G. SnapShot: control of flowering in Arabidopsis. Cell 141, 550-550 (2010).

14. Song, Y. H., Ito, S. \& Imaizumi, T. Flowering time regulation: photoperiod- and temperature-sensing in leaves. Trends plant Sci. 18, 575-583 (2013).

15. Fernández-Calvo, P. et al. The Arabidopsis bHLH transcription factors MYC3 and MYC4 are targets of JAZ repressors and act additively with MYC2 in the activation of jasmonate responses. Plant cell 23, 701-715 (2011).

16. Hu, Y., Jiang, L., Wang, F. \& Yu, D. Jasmonate regulates the INDUCER OF CBF EXPRESSION-C-REPEAT BINDING FACTOR/DRE BINDING FACTOR1 cascade and freezing tolerance in Arabidopsis. Plant Cell 25, 2907-2924 (2013).

17. Cheng, Z. et al. The bHLH transcription factor MYC3 interacts with the jasmonate ZIM-domain proteins to mediate jasmonate response in Arabidopsis. Mol. Plant 4, 279-288 (2011).

18. Niu, Y., Figueroa, P. \& Browse, J. Characterization of JAZ-interacting bHLH transcription factors that regulate jasmonate responses in Arabidopsis. J. Exp. Bot. 62, 2143-2154 (2011).

19. Jiang, Y., Liang, G., Yang, S. \& Yu, D. Arabidopsis WRKY57 functions as a node of convergence for jasmonic acid- and auxin-mediated signaling in jasmonic acid-induced leaf senescence. Plant Cell 26, 230-245 (2014).

20. Qi, T. et al. The Jasmonate-ZIM-domain proteins interact with the WD-Repeat bHLH/MYB complexes to regulate jasmonate-mediated anthocyanin accumulation and trichome initiation in Arabidopsis thaliana. Plant cell $\mathbf{2 3}$ 1795-1814 (2011).

21. Oh, Y., Baldwin, I. T. \& Galis, I. A Jasmonate ZIM-domain protein NaJAZd regulates floral jasmonic acid levels and counteracts flower abscission in Nicotiana attenuata plants. PloS ONE 8, e57868-e57868 (2013).

22. Song, S. et al. The Jasmonate-ZIM domain proteins interact with the R2R3MYB transcription factors MYB21 and MYB24 to affect jasmonate-regulated stamen development in Arabidopsis. Plant Cell 23, 1000-1013 (2011).

23. Cai, Q. et al. Jasmonic acid regulates spikelet development in rice. Nat. Commun. 5, 3476 (2014)

24. Guo, Q. et al. JAZ repressors of metabolic defense promote growth and reproductive fitness in Arabidopsis. Proc. Natl Acad. Sci. USA 115 E10768-E10777 (2018).

25. Thatcher, L. F. et al. Characterization of a JAZ7 activation-tagged Arabidopsis mutant with increased susceptibility to the fungal pathogen Fusarium oxysporum. J. Exp. Bot. 67, 2367-2386 (2016).

26. Oblessuc, P. R. et al. JAZ4 is involved in plant defense, growth, and development in Arabidopsis. Plant J. 101, 371-383 (2020).

27. $\mathrm{Yu}, \mathrm{X}$. et al. The Jasmonate ZIM-domain protein gene SIJAZ2 regulates plant morphology and accelerates flower initiation in Solanum lycopersicum plants. Plant Sci. 267, 65-73 (2018).

28. Nakamura, Y. et al. Arabidopsis florigen FT binds to diurnally oscillating phospholipids that accelerate flowering. Nat. Commun. 5, 3553 (2014).

29. Li, Q. et al. Wrinkled1 accelerates flowering and regulates lipid homeostasis between oil accumulation and membrane lipid anabolism in Brassica napus. Front. Plant Sci. 6, 1015 (2015).

30. Wang, J. et al. The CmTCP2O gene regulates petal elongation growth in Chrysanthemum morifolium. Plant Sci. 280, 248-257 (2019).

31. Withers, J. et al. Transcription factor-dependent nuclear localization of a transcriptional repressor in jasmonate hormone signaling. Proc. Natl Acad. Sci. USA 109, 20148-20153 (2012).

32. Hori, Y., Kurotani, K, Toda, Y., Hattori, T. \& Takeda, S. Overexpression of the JAZ factors with mutated jas domains causes pleiotropic defects in rice spikelet development. Plant Signal. Behav. 9, e970414-e970414 (2014).

33. Love, M. I., Huber, W. \& Anders, S. Moderated estimation of fold change and dispersion for RNA-seq data with DESeq2. Genome Biol. 15, 550-550 (2014).

34. Wahl, V. et al. Regulation of flowering by Trehalose-6-Phosphate signaling in Arabidopsis thaliana. Science 339, 704-707 (2013).
35. Li, L. et al. QQS orphan gene regulates carbon and nitrogen partitioning across species via NF-YC interactions. Proc. Natl Acad. Sci. USA. 112, 14734-14739 (2015).

36. Teaster, N. D. et al. Overexpression of Fatty Acid Amide Hydrolase induces early flowering in Arabidopsis thaliana. Front. plant Sci. 3, 32-32 (2012).

37. Schmid, M. et al. Dissection of floral induction pathways using global expression analysis. Development 130, 6001 (2003).

38. Kim, S., Soltis, P. S., Wall, K. \& Soltis, D. E. Phylogeny and domain evolution in the APETALA2-like gene family. Mol. Biol. Evolution 23, 107-120 (2005).

39. Wasternack, C. \& Song, S. Jasmonates: biosynthesis, metabolism, and signaling by proteins activating and repressing transcription. J. Exp. Bot. 68, 1303-1321 (2016).

40. Ferrandiz, C., Gu, Q., Martienssen, R. \& Yanofsky, M. F. Redundant regulation of meristem identity and plant architecture by FRUITFULL, APETALA1 and CAULIFLOWER. Development 127, 725 (2000).

41. Pabón-Mora, N., Ambrose, B. A. \& Litt, A. Poppy APETALA1/FRUITFULL orthologs control flowering time, branching, perianth identity, and fruit development. Plant Physiol. 158, 1685-1704 (2012).

42. Zhao, $\mathrm{K}$. et al. Characterization of an APETALA1 and a FRUITFUL-like homolog in chrysanthemum. Sci. Horti. 272, 109518 (2020).

43. Pazhouhandeh, M., Molinier, J., Berr, A. \& Genschik, P. MSI4/FVE interacts with CUL4-DDB1 and a PRC2-like complex to control epigenetic regulation of flowering time in Arabidopsis. Proc. Natl Acad. Sci. USA 108, 3430-3435 (2011).

44. Zhu, Y. et al. Characterization of a novel developmentally retarded mutant $(d r m 1)$ associated with the autonomous flowering pathway in Arabidopsis. Cell Res. 15, 133-140 (2005).

45. Shim, J. S., Kubota, A. \& Imaizumi, T. Circadian clock and photoperiodic flowering in Arabidopsis: CONSTANS is a hub for signal integration. Plant Physiol. 173, 5-15 (2016).

46. Teper-Bamnolker, P. \& Samach, A. The flowering integrator FT regulates SEPALLATA3 and FRUITFULL accumulation in Arabidopsis leaves. Plant cell 17 2661-2675 (2005)

47. Yoo, S. K. et al. CONSTANS Activates SUPPRESSOR OF OVEREXPRESSION OF CONSTANS 1 through FLOWERING LOCUS T to promote flowering in Arabidopsis. Plant Physiol. 139, 770-778 (2005).

48. Chen, Y. et al. De novo transcriptome analysis in Dendrobium and identification of critical genes associated with flowering. Plant Physiol. Biochem. 119, 319-327 (2017)

49. Chen, $X$. et al. Comparative RNA-sequencing-based transcriptome profiling of buds from profusely flowering 'Qinguan' and weakly flowering 'Nagafu no. 2' apple varieties reveals novel insights into the regulatory mechanisms underlying floral induction. BMC Plant Biol. 18, 370-370 (2018).

50. Zhu, L. et al. Regulation of flowering time in chrysanthemum by the R2R3 MYB transcription factor CmMYB2 is associated with changes in gibberellin metabolism. Horti. Res. 7, 96-96 (2020).

51. Wang, J. et al. Transcriptomic and hormone analyses reveal mechanisms underlying petal elongation in Chrysanthemum morifolium 'Jinba'. Plant Mol. Biol. 93, 593-606 (2017).

52. Tamura, K. et al. MEGA5: molecular evolutionary genetics analysis using maximum likelihood, evolutionary distance, and maximum parsimony methods. Mol. Biol. Evolution 28, 2731-2739 (2011).

53. Arnim, A. V. Subcellular localization of GUS- and GFP-tagged proteins in onion epidermal cells. Cold Spring Harb. Protoc. 2007, prot4689 (2007).

54. Murashige, T. \& Skoog, F. A revised medium for rapid growth and bio assays with Tobacco tissue cultures. Physiologia Plant. 15, 473-497 (1962).

55. Langmead, B. \& Salzberg, S. L. Fast gapped-read alignment with Bowtie 2. Nat. Methods 9, 357-359 (2012).

56. Grabherr, M. G. et al. Full-length transcriptome assembly from RNA-Seq data without a reference genome. Nature biotechnology 29, 644-652 (2011).

57. Li, B. \& Dewey, C. N. RSEM: accurate transcript quantification from RNASeq data with or without a reference genome. BMC Bioinforma. 12, 323-323 (2011).

58. Anders, S. \& Huber, W. Differential expression analysis for sequence count data. Genome Biol. 11, R106-R106 (2010).

59. Gu, C. et al. Reference gene selection for Quantitative Real-Time PCR in chrysanthemum subjected to biotic and abiotic stress. Mol. Biotechnol. 49 192-197 (2011).

60. Livak, K. J. \& Schmittgen, T. D. Analysis of relative gene expression data using Real-Time Quantitative PCR and the $2^{-\Delta \Delta C T}$ Method. Methods 25, 402-408 (2001). 